\title{
A LATTICE RENORMING THEOREM AND APPLICATIONS TO VECTOR-VALUED PROCESSES
}

BY

\author{
WILLIAM J. DAVIS, ${ }^{1}$ NASSIF GHOUSSOUB ${ }^{2}$ AND JORAM LINDENSTRAUSS ${ }^{3}$
}

\begin{abstract}
A norm, \|\| , on a Banach space $E$ is said to be locally uniformly convex if $\left\|x_{n}\right\| \rightarrow\|x\|$ and $\left\|x_{n}+x\right\| \rightarrow 2\|x\|$ implies that $x_{n} \rightarrow x$ in norm. It is shown that a Banach lattice has an (order) equivalent locally uniformly convex norm if and only if the lattice is order continuous. This result is used to reduce convergence theorems for (lattice-valued) positive martingales and submartingales to the scalar case.
\end{abstract}

0. Introduction. A norm, \|\| , on a Banach space $E$ is said to have the Kadec-Klee property (sometimes property $(\mathrm{H})$ ) if whenever $x_{n} \rightarrow x$ weakly and $\left\|x_{n}\right\| \rightarrow\|x\|$, then $x_{n} \rightarrow x$ strongly. The aim of this paper is to show that if $E$ has an equivalent Kadec-Klee norm then one may obtain convergence theorems for $E$-valued random processes $\left(X_{n}\right)$ whenever $\left\|X_{n}\right\|$ is a well-known real-valued convergent process and whenever the limit can be identified in the Banach space. For instance, the classical Kadec renorming theorem for separable Banach spaces gives the convergence of vector-valued martingales, uniform amarts and additive processes (ergodic theorem) since their norms are real-valued submartingales, amarts and subadditive processes respectively while the identification of the limit requires the RadonNikodym property on the space for the first two processes, the limit of the third process exists in any Banach space.

If now, we consider positive submartingales and subadditive processes valued in a Banach lattice, one needs that the equivalent Kadec-Klee norm be also a lattice norm in order to conclude that the norms of these processes are real-valued submartingales and subadditive processes respectively. §I deals with the existence of such a lattice renorming while in §II we show how this leads to a unified approach for proving the almost sure convergence of the processes mentioned above.

I. Renorming order continuous lattices. A norm, \|\| , on a Banach space $E$ is said to be locally uniformly convex if $\left\|x_{n}\right\| \rightarrow\|x\|$ and $\left\|x+x_{n}\right\| \rightarrow 2\|x\|$ imply that $x_{n} \rightarrow x$ strongly. This notion is clearly stronger than the Kadec-Klee property.

Received by the editors October 15, 1979 and, in revised form, February 11, 1980; presented to the Society, San Antonio, January 1980.

AMS (MOS) subject classifications (1970). Primary 46B99, 60G99.

Key words and phrases. Banach lattice, local uniform convexity, renorming, vector-valued processes, martingales, submartingales, ergodic theorem.

${ }^{1}$ Partially supported by NSF MCS78-02194.

${ }^{2}$ This work was partially supported by NSF Grant MCS77-04909.

${ }^{3}$ Supported in part by NSF Grant MCS78-02194. 
Kadec showed that every separable Banach space has an equivalent locally uniformly convex norm [2, p. 176]. Trojanski extended this to all weakly compactly generated spaces [5, p. 164]. The difficulty with the known renormings of this type in case $E$ is a lattice is that they use many seminorms of the form $\operatorname{dist}(x, F)$ where $F$ is a finite-dimensional subspace of $E$. These are almost never lattice seminorms. The purpose of this section is to overcome this difficulty.

Let $E$ be a Banach space which is also a linear lattice with partial order denoted by $<$. That is, for $x, y \in E$, there exists an element $x \vee y$ in $E$, the least upper bound of $x$ and $y$. Further, if $0<x<y$ and $a>0,0<a x<a y$, and for $a<0$, $0>a x>a y$. For any $x \in E$, let $|x|=x \vee 0-x \wedge 0$, where $x \wedge y$ denotes the greatest lower bound of $x$ and $y$, The norm on $E$ is a lattice norm if $|x| \leqslant|y|$ implies $\|x\| \leqslant\|y\|$.

Recall that a lattice is said to be order continuous if every order-convergent filter is norm convergent. For example, $L_{1}$ is order continuous and $C[0,1]$ is not. In fact, as we shall see, the choice of $L_{1}$ as a prototype of an order continuous lattice is good, since our renorming will depend upon the existence of the same type of renorming for $L_{1}$. We show here that a Banach lattice is order continuous if and only if it has a lattice-equivalent locally uniformly convex lattice norm.

We need some notation first. Let $(\Omega, \mathscr{F}, P)$ be a probability space. For any $f \in L_{1}(\Omega, \mathcal{F}, P)$, let $\tilde{f}(t)=\sup _{P(A) \leqslant t} \int_{A}|f| d P$. Notice that if $f^{*}$ denotes the decreasing rearrangement of $f$, then $\tilde{f}(t)=\int_{0}^{t} f^{*}(s) d s$, so the map $f \rightarrow \tilde{f}$ is subadditive and maps $L_{1}(\Omega, P)$ into each $L_{p}[0,1]$ space boundedly.

The basic facts about lattices which we use are available in the books [12] and [16].

Proposition I.1. Let $\left\{f_{n}\right\}$, f be in $L_{1}(\Omega, P)$, so that $\tilde{f}_{n} \rightarrow \tilde{f}$ a.s. and $\widetilde{f+f_{n}} \rightarrow 2 \tilde{f}$ a.s. Then $f_{n} \rightarrow f$ in norm.

Proof. Since $\tilde{f}_{n}(t) \rightarrow \tilde{f}(t)$ a.s., and since $\tilde{f}(t) \rightarrow 0$ as $t \rightarrow 0$, we see that the original sequence $\left\{f, f_{n}\right\}$ is equi-integrable. That is, for any $\varepsilon>0$ there is $\delta>0$ so that $P(A)<\delta$ implies $\int\left|f_{n}\right| \chi_{A} d P<\varepsilon$ and $\int|f| \chi_{A} d P<\varepsilon$. This allows us to show that $\tilde{f}_{n} \rightarrow \tilde{f}$ uniformly on $[0,1]$. Let $\delta=\delta(\varepsilon / 3)$ from above and choose a finite set $\left\{x_{1}, \ldots, x_{m}\right\}$ in $[0,1]$ so that, for $x \in[0,1], \min \left|x-x_{i}\right|<\delta / 3$, and such that $\tilde{f}_{n}\left(x_{i}\right) \rightarrow \tilde{f}\left(x_{i}\right)$ for $i=1,2, \ldots, m$. Let $N$ be large enough so that $n \geqslant N$ implies $\left|\tilde{f}_{n}\left(x_{i}\right)-\tilde{f}\left(x_{i}\right)\right|<\varepsilon / 3$. We have, then, that for $x$ in $[0,1]$ and $\left|x-x_{i}\right|<\delta, \mid \tilde{f}_{n}(x)-$ $\tilde{f}(x)|\leqslant| \tilde{f}_{n}(x)-\tilde{f}_{n}\left(x_{i}\right)|+| \tilde{f}_{n}\left(x_{i}\right)-\tilde{f}\left(x_{i}\right)|+| \tilde{f}(x)-\tilde{f}\left(x_{i}\right) \mid$. The first and last terms are $<\varepsilon / 3$ by the equi-integrability, so the sequence is converging uniformly. Without loss of generality we may assume that $f \geqslant 0$. We first also make the further assumption that $f_{n} \geqslant 0$ for each $n$. The distribution function of $f, P[f \geqslant \lambda]$, is left continuous, so that for each $\lambda, \alpha>0$, there is $\beta>0$ so that $P[f \geqslant \lambda-\beta]$ $-P[f \geqslant \lambda]<\alpha$. For notational purposes, let $A_{\lambda}=[f \geqslant \lambda]=B_{\lambda}^{c}$ and $A_{n, \lambda}=\left[f_{n}\right.$ $\geqslant \lambda]=B_{n, \lambda}^{c}$. Fix $\lambda>0$. Now let $\varepsilon>0$ and let $\delta$ be as before. Let $\eta>0$ be small enough so that $P\left(A_{\lambda-\eta} \cap B_{\lambda}\right)<\delta / 4$ and $\eta<\delta / 4$. Next, let $0<\gamma<\eta^{2}$. Select $n$ large enough so that $\left|\widetilde{f_{n}+f}\left(P\left(A_{\lambda}\right)\right)-2 \tilde{f}\left(P\left(A_{\lambda}\right)\right)\right|<\gamma / 2$ and $\mid \tilde{f}_{n}\left(P\left(A_{\lambda}\right)\right)-$ $\tilde{f}\left(P\left(A_{\lambda}\right)\right) \mid<\gamma / 2$. Choose a set $A$ with $P(A)=P\left(A_{\lambda}\right)$ so that $\int_{A}\left(f_{n}+f\right) d P>$ $\widetilde{f_{n}+f}\left(P\left(A_{\lambda}\right)\right)-\gamma / 2$. This yields 


$$
\begin{aligned}
2 \int_{A_{\lambda}} f d P-\gamma & <\int_{A} f d P+\int_{A} f_{n} d P \leqslant \int_{A} f d P+\tilde{f}_{n}\left(P\left(A_{\lambda}\right)\right) \\
& \leqslant \int_{A} f d P+\tilde{f}\left(P\left(A_{\lambda}\right)\right)+\gamma / 2=\int_{A} f d P+\gamma / 2+\int_{A_{\lambda}} f d P .
\end{aligned}
$$

That is, $\int_{A} f d P>\int_{A_{\lambda}} f d P-2 \gamma$. Next we have

$$
\begin{aligned}
\int_{A} f d P & =\int_{A \cap A_{\lambda-\eta}} f d P+\int_{A \cap B_{\lambda-\eta}} f d P \\
& \leqslant \tilde{f}\left(P\left(A \cap A_{\lambda-\eta}\right)\right)+(\lambda-\eta) P\left(A \cap B_{\lambda-\eta}\right) \\
& =\tilde{f}\left(P(A)-P\left(A \cap B_{\lambda-\eta}\right)\right)+(\lambda-\eta) P\left(A \cap B_{\lambda-\eta}\right) .
\end{aligned}
$$

Putting these together, we get

$$
\begin{aligned}
\lambda P\left(A \cap B_{\lambda-\eta}\right)-2 \gamma & \leqslant \tilde{f}\left(P\left(A_{\lambda}\right)\right)-\tilde{f}\left(P\left(A_{\lambda}\right)-P\left(A \cap B_{\lambda-\eta}\right)\right)-2 \gamma \\
& \leqslant(\lambda-\eta) P\left(A \cap B_{\lambda-\eta}\right) .
\end{aligned}
$$

Thus $P\left(A \cap B_{\lambda-\eta}\right) \leqslant 2 \gamma / \eta<2 \eta$. Finally, $A=\left(A \cap A_{\lambda}\right) \cup\left(A \cap B_{\lambda} \cap A_{\lambda-\eta}\right) \cup$ $\left(A \cap B_{\lambda-\eta}\right)$ and $P(A)=P\left(A_{\lambda}\right)$ give $P\left(A \cap A_{\lambda}\right) \leqslant P(A)-\delta$. Similarly, we get that $P\left(A \cap B_{n, \lambda-\eta}\right)<\delta$, so that $P\left(A_{\lambda} \cap B_{n, \lambda-\eta}\right)<2 \delta$. What we have shown is that for all $\lambda>0$ and $\eta>0, P\left[f \geqslant \lambda, f_{n}<\lambda-\eta\right] \rightarrow 0$ as $n \rightarrow \infty$. It is a routine exercise to show that this implies that $P\left[f_{n}<f-\eta\right] \rightarrow 0$ as $n \rightarrow \infty$. Once again, fix $\eta, \eta_{1}>0$. Then $\tilde{f}_{n}(1)=\int f_{n} d P=\int_{\left[f_{n}>f+\eta\right]} f_{n} d P+\int_{\left[f_{n}<f+\eta\right]} f_{n} d P$ so

$$
\begin{aligned}
\tilde{f}_{n}(1) & \geqslant \int_{\left[f_{n}>f+\eta\right]} f_{n} d P+\int_{\left[f-\eta_{1}<f_{n}<f+\eta\right]} f_{n} d P+\int_{\left[f_{n}<f-\eta_{1}\right]} f_{n} d P \\
& \geqslant \eta P\left[f_{n} \geqslant f+\eta\right]+\int_{\left[f_{n}>f-\eta_{1}\right]} f d P-\eta_{1}+\int_{\left[f_{n}<f-\eta_{1}\right]} f_{n} d P .
\end{aligned}
$$

As $n \rightarrow \infty$, the left-hand side tends to $\tilde{f}(1)$, the second term on the right tends to $\tilde{f}(1)$ and the last term on the right tends to zero. Thus, $\lim \sup P\left[f_{n}>f+\eta\right]<$ $\eta_{1} / \eta$. Since $\eta_{1}$ is chosen arbitrarily, this then yields $P\left[f_{n}>f+\eta\right] \rightarrow 0$ as $n \rightarrow \infty$. Therefore, we have $f_{n} \rightarrow f$ in measure. Since we already knew that $\left\{f_{n}\right\}$ is equi-integrable, this forces $f_{n} \rightarrow f$ in $L_{1}$. Without assuming $f_{n}>0$, we have shown that $\left|f_{n}\right| \rightarrow f$ in $L_{1}$ norm. It is easy to see that this together with $\widetilde{f_{n}+f} \rightarrow 2 \tilde{f}$ forces $f_{n} \rightarrow f$ in $L_{1}$ norm, completing the proof.

Now we are ready to renorm $L_{1}(\Omega, P)$.

TheOREM I.1. If $(\Omega, P)$ is a probability space, then $L_{1}(\Omega, P)$ has an equivalent locally uniformly convex lattice norm.

Proof. Setting $\|f\|_{1}=\|f\|_{L_{1}(\Omega, P)}$ and $\|f\|_{2}=\|\tilde{f}\|_{\left.L_{2}(0,1]\right)}$, we let

$$
\|f\|=\left(\|f\|_{1}^{2}+\|f\|_{2}^{2}\right)^{1 / 2} \text {. }
$$

It is clear that this is a lattice norm, order equivalent to the $L_{1}(\Omega, P)$ norm. Let $f_{n}, f$ be in $L_{1}(\Omega, P)$ so that $\left\|f_{n}\right\| \rightarrow\|f\|$ and $\left\|f_{n}+f\right\| \rightarrow 2\|f\|$ as $n \rightarrow \infty$. Using the triangle inequality, we see that

$$
\left\{\left(\|f\|_{1}+\left\|f_{n}\right\|_{1}\right)^{2}+\left(\|f\|_{2}+\left\|f_{n}\right\|_{2}\right)^{2}\right\}^{1 / 2} \rightarrow 2\left(\|f\|_{1}^{2}+\|f\|_{2}^{2}\right)^{1 / 2}
$$


so that, in particular, we must have $\left\|f_{n}\right\|_{i} \rightarrow\|f\|_{i}$ and $\left\|f_{n}+f\right\|_{i} \rightarrow 2\|f\|_{i}$ for $i=1,2$ as $n \rightarrow \infty$. Since $L_{2}([0,1])$ is uniformly convex, these force $\tilde{f}_{n} \rightarrow \tilde{f}$ in $L_{2}([0,1])$, and hence in measure. Therefore, an arbitrary subsequence of $\tilde{f}_{n}$ has a subsequence converging to $\tilde{f}$ almost everywhere. Applying Proposition I.1, we have the desired conclusion.

COROLLARY I.1. $L_{1}([0,1])$ has an order-equivalent, rearrangement-invariant, locally uniformly convex norm.

Proof. If $(\Omega, P)=([0,1], \mu)$, the above norm is rearrangement invariant.

We have now done most of the work required to prove the renorming theorems for general order-continuous lattices. There is just one ingredient left to be built into the norm - a uniform integrability condition in the lattice norm. Suppose that $E$ is an order-continuous lattice with a weak order unit (that is, $e \in E^{+}$such that $e \wedge|f|=0$ implies $f=0$ ). Then we may consider this lattice as a function space on some $(\Omega, P)$ with the property that $L_{\infty}(\Omega, P) \subset E \subset L_{1}(\Omega, P)$ and so that for $f \in E,\|f\|_{1} \leqslant\|f\|_{E}$. See, for example, [12, 1.b.14]. The order continuity of the norm forces $\lim _{P(A) \rightarrow 0}\left\|f \chi_{A}\right\|_{E} \rightarrow 0$ for each $f \in E$. Thus, if we let $\Psi_{n}(f)=$ $\sup _{P(A) \leqslant 1 / n}\left\|f \chi_{A}\right\|_{E}$, and if $\Psi_{n}\left(f_{k}\right) \rightarrow \Psi_{n}(f)$ as $k \rightarrow \infty$ for each $n$, then the sequence $\left\{f_{k}\right\}$ must have the property that for all $\varepsilon>0$ there is $\delta>0$ so that $P(A)<\delta$ implies $\left\|f_{k} \chi_{A}\right\|_{E}<\varepsilon$, for all $k$. One should notice that each of the norms $\Psi_{n}$ is consistent with the ordering in the lattice, and that, when it makes sense, each is rearrangement invariant as well.

We denote by $\tilde{c}_{0}$ the Banach lattice $c_{0}$ equipped with its locally uniformly convex lattice norm constructed by Day [5, p. 94]. The following is the main result of this section.

THEOREM I.2. A Banach lattice, $E$, has an order-continuous norm if and only if it has an equivalent locally uniformly convex lattice norm.

Proof. Assume first that $E$ is order continuous. By Kakutani's theorem, $E=$ $\Sigma_{\alpha \in \Gamma} \bigoplus E_{\alpha}$ where each $E_{\alpha}$ is a band with weak order unit $e_{\alpha}$ [12]. In particular for each $f \in E, f=\sum_{\alpha \in \Gamma} f_{\alpha}$, and each of the maps $f \rightarrow f_{\alpha}$ is a (positive) band projection. We first renorm each $E_{\alpha}$.

As above, for each $\alpha$, there is a probability space $\left(\Omega_{\alpha}, P_{\alpha}\right)$ so that $L_{1}\left(\Omega_{\alpha}, P_{\alpha}\right) \supset$ $E_{\alpha} \supset L_{\infty}\left(\Omega_{\alpha}, P_{\alpha}\right)$. For this part of the argument, let us ignore the subscripts, $\alpha$, that occur. Let $\|\cdot\|_{E}$ denote the original norm on the lattice, $\|\cdot\| \|_{1}$, the norm on $L_{1}(\Omega, P)$ constructed in Theorem $I .1$ and $\Psi_{n}(\cdot)$, the norms defined above. Define the new norm on $E$ by $\||f|\|=\left\|\left(\|f\|_{E},\|\| f \|_{1}, \Psi_{1}(f), \Psi_{2}(f), \ldots\right)\right\|_{\tilde{c}_{0}}$. It is immediate that $\|\cdot\| \|$ is a lattice norm equivalent to $\|\cdot\|_{E}$. Also, as before, if $\left\|\left|f_{n}\||\rightarrow|\| f\right|||\right.$ and $\|\left|f+f_{n}\left\|\left|\rightarrow 2\||| f \mid\|\right.\right.\right.$, then, by the nature of the norm in $\tilde{c}_{0}$, this forces $\left\|f_{n}\right\|_{E} \rightarrow\|f\|_{E},\left\|f_{n}\right\|\left\|_{1} \rightarrow\right\|\|f\|_{1}$ and $\Psi_{k}\left(f_{n}\right) \rightarrow \Psi_{k}(f)$ when $n \rightarrow \infty$ for each $k$. In particular, we get from Theorem I.1 that $f_{n} \rightarrow f$ in the $L_{1}(\Omega, P)$ norm. This forces $f_{n} \rightarrow f$ in measure. Set $A_{n}=\left[\left|f_{n}-f\right|<\varepsilon\right]$.

$\left\|f_{n}-f\right\|_{E} \leqslant\left\|\left(f_{n}-f\right) \chi_{A_{n}}\right\|_{E}+\left\|\left(f-f_{n}\right) \chi_{A_{n}^{c}}\right\|_{E} \leqslant \varepsilon\left\|\chi_{A_{n}}\right\|_{E}+\left\|f \chi_{A_{n}}\right\|_{E}+\left\|f_{n} \chi_{A_{n}}\right\|_{E}$.

The convergence of $\Psi_{k}\left(f_{n}\right)$ for each $k$ (to $\Psi_{k}(f)$ ) has forced the lattice norm 
uniform integrability of the sequence, so the terms $\left\|f \chi_{A_{n}^{c}}\right\|$ and $\left\|f_{n} \chi_{A_{n}^{c}}\right\|$ approach 0 as $n \rightarrow \infty$. This completes the proof for this case.

To extend this to the general case, let $\|\mid \cdot\|_{\alpha}$ denote the norm just constructed on $E_{\alpha}$ for each $\alpha$. The rest of the renorming follows the lines of the proof of Troyanski's renorming of WCG spaces as presented in Day's book [4, p. 162]. Let $Q_{\alpha}$ denote the band projection of $E$ onto $E_{\alpha}$. For any finite set $A \subset \Gamma$, let $E_{A}(f)=\left\|\left(I-\sum_{\alpha \in A} Q_{\alpha}\right) f\right\|_{E}$. This is again a lattice seminorm. Let $G_{A}(f)=$ $(\operatorname{card} A) \sum_{\alpha \in A}\left\|Q_{\alpha} f\right\| \|_{\alpha}+E_{A}(f)$, and let $G_{n}(f)=\sup \left\{G_{A}(f) \mid \operatorname{card} A=n\right\}$. If we now let $S$ be the index set $\{\{0\}, \Gamma, \mathbf{N}\}$, and define $\Phi_{0}(f)=\|f\|_{E}, 2^{n} \Phi_{n}(f)=G_{n}(f)$ for $n \in \mathbf{N}$ and $\Phi_{\alpha}(f)=\left\|Q_{\alpha} f\right\|$, for all $\alpha \in \Gamma$, then $f \rightarrow\left\{\Phi_{s}(f)\right\}_{s \in S}$ takes $E$ to $\tilde{c}_{0}(S)$. Thus, the equivalent lattice norm on $E$ is defined to be $\|f\| \|=$ $\left\|\left\{\Phi_{s}(f)\right\}\right\|_{\tilde{c}_{0}(S)}$. Again, $\left\|f_{j}\right\|\|\rightarrow\| f \| \mid$ and $\left\|\mid f+f_{j}\right\| \rightarrow \rightarrow 2\|f\| \|$ forces $\Phi_{s}\left(f_{j}\right) \rightarrow \Phi_{s}(f)$ and $\Phi_{s}\left(f+f_{j}\right) \rightarrow 2 \Phi_{s}(f)$ for all $s$ in $S$. By the preceding paragraph, we see that $Q_{\alpha} f_{j} \rightarrow Q_{\alpha} f$ in $E_{\alpha}$ for each $\alpha$. By the definition of $G_{n}$, for any set $A$ with cardinality $k$, we have $k \Sigma_{\alpha \in A}\left\|Q_{\alpha} f\right\|_{\alpha}+E_{A}(f) \leqslant G_{k}(f)$. Thus, $E_{A}\left(f_{j}\right) \leqslant G_{k}\left(f_{j}\right)-$ $k \sum_{\alpha \in A} \mid\left\|Q_{\alpha} f_{j}\right\|$. . Now let $\varepsilon>0$, and choose $A$ so that $G_{k}(f)-G_{A}(f)<\varepsilon$. Since $G_{k}\left(f_{j}\right) \rightarrow G_{k}(f)$ and $\left\|Q_{\alpha} f_{j}\right\|_{\alpha} \rightarrow\left\|Q_{\alpha} f\right\|_{\alpha}$ as $j \rightarrow \infty$, we may choose $j$ large enough so that $E_{A}\left(f_{j}\right) \leqslant E_{A}(f)+2 \varepsilon$. The goal now is to make $E_{A}(f)$ small for the proper choice of $k$.

Choose a set $A_{1}$ so that $E_{A_{1}}(f)<\varepsilon$ and so that $\min _{\alpha \in A_{1}}\left\|\mid Q_{\alpha} f\right\|_{\alpha}=a>0$. Let $A_{2}=A_{1} \cup\left\{\beta\left|\left\|Q_{\beta} f \mid\right\|_{\beta} \geqslant a\right\}\right.$. Since $\left\{\left\|\left|Q_{\alpha} f\right|\right\|_{\alpha}\right\} \in c_{0}(\Gamma)$, there is $\Delta>0$ so that for $\beta \notin A_{2},\left\|Q_{\beta} f\right\|_{\beta}<a-\Delta$. Let $k \geqslant\left\{\operatorname{card} A_{2},(\varepsilon+\|f\|) / \Delta\right\}$, and select $A$ with cardinality $k$ so that $G_{k}(f)-G_{A}(f)<\varepsilon$. Suppose, $A_{2} \underline{E} A$ and let $\beta \in A_{2} \backslash A$, $\gamma \in A \backslash A_{2}$. Let $B=A \cup\{\beta\} \backslash\{\gamma\}$. Then

$$
\begin{aligned}
G_{k}(f)-E_{A}(f)- & k \sum_{\alpha \in A}\left\|Q_{\alpha} f\right\|_{\alpha} \\
& \geqslant E_{B}(f)+k \sum_{\alpha \in B}\left\|\mid Q_{\alpha} f\right\|_{\alpha}-E_{A}(f)-k \sum_{\alpha \in A}\left\|Q_{\alpha} f\right\| \\
& \geqslant-E_{A}(f)+k \Delta \geqslant-\|f\|_{E}+k \Delta \geqslant \varepsilon,
\end{aligned}
$$

which is a contradiction. Thus, $A \supset A_{2} \supset A_{1}$ and so $E_{A}(f) \geqslant E_{A_{1}}(f)<\varepsilon$. We have, then, that for this choice of $k$ and $A$, and for $j$ sufficiently large, $E_{A}\left(f_{j}\right)<3 \varepsilon$. Finally, $\left\|f-f_{j}\right\|_{E} \leqslant E_{A}(f)+E_{A}\left(f_{j}\right)+k \Sigma_{\alpha \in A}\left\|Q_{\alpha}\left(f-f_{j}\right)\right\|_{\alpha}$. Each term of this finite sum tends to zero as $j \rightarrow \infty$, so we get for arbitrary $\varepsilon$, a $j_{0}$ so that $j \geqslant j_{0}$ implies $\left\|f-f_{j}\right\|_{E}<4 \varepsilon$, and we are done with the renorming.

If $E$ is not order continuous, then $E$ contains positive, norm one vectors $\left\{x_{n}\right\}$ converging weakly to zero, and $x$ such that $x_{n} \leqslant x$ for all $n$. Thus, $0 \leqslant x-x_{n}<x$ and we have $\left\|x-x_{n}\right\| \rightarrow\|x\|$, but $x-x_{n} \nrightarrow x$ so the norm cannot be locally uniformly convex and cannot even be Kadec-Klee.

We now write a more general version of Corollary I.1. Since the proofs are nearly identical with those of above, we do not include them here.

COROLlaRY I.2. If $E$ is a separable, rearrangement invariant function space on $[0,1]$, it has an equivalent, rearrangement invariant locally uniformly convex lattice norm. 
Corollary I.3. Suppose that $E$ is a complemented subspace of a Banach lattice, $F$, and suppose that $E$ does not contain a subspace isomorphic to $c_{0}$. Then $E$ has an equivalent locally uniformly convex norm.

Proof. By [9], $E$ embeds into a lattice $F_{1}$ which does not contain $c_{0}$. Any such $F_{1}$ has order-continuous norm, and so may be renormed as above. This renorming is as desired on $E$.

II. Almost sure convergence of vector-valued processes. In this section, we will use the following version of the renorming theorem:

If $E$ is a separable order continuous Banach lattice, then it admits an equivalent lattice norm which is Kadec-Klee with respect to a countable and norming subset $D$ of the positive cone of the dual (i.e. $\|x\|=\sup \left\{x^{*}(|x|) ; x^{*} \in D\right\}$ for every $x \in E$ ).

Now let $(\Omega, \mathcal{F}, P)$ be a probability space and $\left(S_{n}\right)$ a sequence of $E$-valued Bochner integrable random variables. Since the $S_{n}$ 's are almost separably valued, we may and shall assume without loss of generality that $E$ is separable. If $E$ has also an order continuous norm, it will be equipped with the norm \|\| and the set $D$ mentioned above.

If $\left(\mathscr{F}_{n}\right)_{n}$ is a sequence of sub- $\sigma$-algebras increasing to $\mathscr{F}_{\text {, we say that }}\left(S_{n}, \mathscr{F}_{n}\right)$ is a submartingale if $\left(S_{n}\right)$ is $\left(\mathscr{F}_{n}\right)$-adapted and if $E^{\mathscr{F}_{n}}\left[X_{n+1}\right]>X_{n}$ a.e. for each $n>0$. The basic facts about vector measures which we use are available in the book [6].

In the following, we will make constant use of the following lemma proved in $[13$, p. 103].

Lemma II.1. Let I be a denumerable set, and for each $i \in I$, let $\left(S_{n}^{i}\right)_{n}$ be a real submartingale. If $\sup _{n} \int \sup _{i}\left(S_{n}^{i}\right)^{+}<\infty$, then

(i) for every $i \in I,\left(S_{n}^{i}\right)$ converges a.e. to a limit $S_{\infty}^{i}$,

(ii) the submartingale $\left(\sup _{i} S_{n}^{i}\right)$ converges a.e. to $\sup _{i} S_{\infty}^{i}$.

THEOREM II. 1. [8]. If $E$ is a Banach lattice with the Radon-Nikodym property, then every $E$-valued $L^{1}$-bounded positive submartingale converges strongly a.e.

Proof. For every $A \in \cup_{n} \mathscr{F}_{n},\left(\int_{A} S_{n}\right)$ is an increasing sequence (after a certain rank) which is norm bounded; hence it converges to $\mu(A)$. The vector measure $\mu$ is of bounded variation, hence it is strongly additive. By the Caratheodory-HahnKluvanek extension theorem [6, p. 27], $\mu$ can be extended to a countably additive measure on $\mathscr{F}$, absolutely continuous with respect to $P$ and of finite variation. Thus, by the Radon-Nikodym property, there exists an $E$-valued Bochner integrable random variable $S_{\infty}$ such that $\lim _{n} \int_{A} S_{n}=\int_{A} S_{\infty}$ for every $A \in \mathscr{F}$.

For each $f \in D, f\left(S_{n}\right)$ is a real-valued submartingale which converges necessarily to $f\left(S_{\infty}\right)$ outside $\Omega_{f}$ with $P\left(\Omega_{f}\right)=0$. On the other hand, we have for every $x \in E_{+}$, $\|x\|=\sup _{f \in D} f(x)$, hence Lemma II.1 yields that $\left\|S_{n}\right\|=\sup _{f \in D} f\left(S_{n}\right)$ converges to $\sup _{f \in D} f\left(S_{\infty}\right)=\left\|S_{\infty}\right\|$ outside $\Omega_{0}$ with $P\left(\Omega_{0}\right)=0$. The Kadec-Klee property of the norm gives the strong convergence of $\left(S_{n}\right)$ outside $\Omega_{0} \cup \cup_{f \in D} \Omega_{f}$.

REMARKS. (1) The classical Kadec renorming theorem (mentioned in the introduction) gives a relatively easy proof of the well-known convergence theorem for 
vector-valued martingales [3]. If $E$ is a Banach space with the Radon-Nikodym property and $\left(S_{n}\right)$ is an $E$-valued $L^{1}$-bounded martingale, then the same reasoning as in Theorem II.1 gives a random variable $S_{\infty}$ such that $\lim _{n} \int_{A} S_{n}=\int_{A} S_{\infty}$ for every $A \in F$. Lemma II.1 applies to give that $\left\|S_{n}\right\|=\sup _{f \in D}\left|f\left(S_{n}\right)\right|$ converges a.e. to $\left\|S_{\infty}\right\|$. The convergence follows from the Kadec-Klee property of the norm.

(2) A similar proof can be given for uniform amarts, since it may be shown [1] that $\left\|S_{n}\right\|$ is then a real amart and if the space has the R.N.P., it converges necessarily a.e. to $\left\|S_{\infty}\right\|$.

THEOREM II.2. If $E$ has an order-continuous norm, and $\left(S_{n}\right)$ is a submartingale such that $0 \leqslant S_{n} \leqslant E^{\mathscr{G}_{n}}[S]$ for some random variable $S$, then $\left(S_{n}\right)$ converges strongly a.e.

Proof. Let $\Omega_{0}$ be the null set where $E^{\mathscr{F}_{n}}[S]$ does not converge. Without loss of generality, we may assume that the limit is $S$. (Otherwise, replace $S$ by $E^{\mathscr{G}}(S)$ where $\mathcal{F}=\bigvee \mathscr{F}_{n}$.) Clearly $S \leqslant S_{n} \vee S \leqslant E^{\mathscr{F}_{n}}[S] \bigvee S$, and hence $\lim S_{n} \vee S=S$ outside $\Omega_{0}$. For each $f \in D$, there is an $\Omega_{f}$ with $P\left(\Omega_{f}\right)=0$ such that $f\left(S_{n}\right)$ converges on $\Omega \backslash \Omega_{f}$. Since $S_{n} \vee S+S_{n} \wedge S=S_{n}+S$, we get that off the set $\Omega_{1}=\Omega_{0} \cup$ $\cup_{D} \Omega_{f}, \lim f\left(S_{n} \wedge S\right)$ exists for every $f \in D$. Since order intervals in an ordercontinuous lattice are weakly compact, we deduce that $S_{n} \wedge S$ converges weakly off $\Omega_{1}$. Therefore, $S_{n}$ converges weakly to some random variable, $S_{\infty}$.

Again, Lemma II.1 applies and gives that $\left\|S_{n}\right\|$ converges $\left\|S_{\infty}\right\|$ a.e. and the theorem follows by the Kadec-Klee property.

COROllaRY II.1. If $E$ has an order-continuous norm, let $\mu$ be an E-valued vector measure order bounded by two differentiable vector measures; that is there exists $X$ and $Y$ in $L^{1}[E]$ such that for every $A \in \mathcal{F}$

$$
\int_{A} Y d P \leqslant \mu(A) \leqslant \int_{A} X d P .
$$

Then, there exists $Z$ in $L^{1}[E]$ such that $Y \leqslant Z \leqslant X$ a.e. and $\mu(A)=\int_{A} Z d P$ for every $A \in \mathcal{F}$.

Proof. Clearly, we may assume that $0 \leqslant \mu(A) \leqslant \int_{A} X d P$ for every $A \in \mathscr{F}$. We associate now to $\mu$, a positive martingale as follows. Assuming the $\sigma$-field separable, we may construct an increasing sequence of $\sigma$-fields $\left(\mathscr{F}_{n}\right)_{n}$ generated by finite partitions $\left\{B_{n}^{p} ; 1 \leqslant p \leqslant p_{n}\right\}$ and generating the $\sigma$-field $\mathscr{F}$. Set

$$
Z_{n}=\sum_{1<p<p_{n}} \frac{\mu\left(B_{n}^{p}\right)}{P\left(B_{n}^{p}\right)} \chi_{B_{n}^{p}} \quad \text { with } \frac{\mu\left(B_{n}^{p}\right)}{P\left(B_{n}^{p}\right)}=0 \text { if } P\left(B_{n}^{p}\right)=0 \text {. }
$$

The sequence $\left(Z_{n}\right)$ is an $\mathscr{F}_{n}$-martingale verifying $0 \leqslant Z_{n} \leqslant E^{\mathscr{F}_{n}}[X]$. By the preceding theorem, $Z_{n}$ converges to $Z$. Clearly, $0 \leqslant Z \leqslant X$ and $\mu(A)=\int_{A} Z d P$ for every $A \in \mathscr{F}$.

In case the field is not separable, we note that, by the first part of the proof, for every separable sub- $\sigma$-field, $\mathscr{F}^{1}$ of $\mathscr{F},\left\{\mu(A) \mid A \in \mathscr{F}^{1}\right\}$ is totally bounded. Hence, $\{\mu(A) \mid A \in \mathscr{F}\}$ is also totally bounded, and thus, separable. We may therefore assume that $E$ is separable. Let $D \subset E^{*}$ be a countable set as usual. The result 
follows by virtue of the fact that it is valid for the separable sub- $\sigma$-field with respect to which all $d(f \circ \mu) / d P$ are measurable for all $f \in D$.

The following corollary can be alternately proved as a corollary of Theorem III.2.18 in [6].

Corollary II.2. If $E$ has an order-continuous norm and $X$ is an $E$-valued, Bochner integrable random variable then

$$
\int_{A} X^{+} d P=\sup _{B \in \mathscr{F}} \int_{A \cap B} X d P \text { for every } A \in \mathscr{F} .
$$

Proof. Since $E$ has an order-continuous norm, $\mu^{+}(A)=\sup _{B \in \mathscr{F}} \int_{A \cap B} X d P$ exists and is a countably additive measure verifying for every $A \in \mathscr{F}, 0 \leqslant \mu^{+}(A)$ $\leqslant \int_{A} X^{+} d P$. Corollary II.1 applies to give $Z$ in $L^{1}[E]$ such that $0 \leqslant Z \leqslant X^{+}$and $\mu^{+}(A)=\int_{A} Z d P$ for all $A \in \mathcal{F}$. The same reasoning applied to $\mu^{-}$shows that there exists $Y, 0 \leqslant Y \leqslant X^{-}$and $\mu^{-}(A)=\int_{A} Y d P$. We get that $Z-Y=X^{+}-$ $X^{-}$a.e. and since both of them are orthogonal, we get that $Z=X^{+}$and $Y=X^{-}$.

Corollary II.3 (Vector-valued Strassen Theorem). If $F$ is a vector space, $E$ an order-continuous Banach lattice, $\Psi: F \rightarrow L^{1}[E]$ is sublinear and $\varphi: F \rightarrow E$ is linear such that $\varphi(x) \leqslant \int \Psi(x) d P$ for all $x \in F$, then there exists a linear operator $T: F \rightarrow L^{1}[E]$ such that $T(x) \leqslant \Psi(x)$ and $\varphi(x)=\int T(x) d P$ for all $\dot{x}$ in $F$.

Proof. Let $M$ be the space of all $F$-valued measurable simple functions. The vector space $F$ can be identified to the subspace of all constant functions from $\Omega$ to $F$. Obviously, the mapping $\theta: M \rightarrow E$ defined by $\theta(f)=\int \Psi(f(w))(w) d P$ is sublinear, thus, the generalized Hahn-Banach theorem [14, p. 109] gives a linear mapping $\Phi: M \rightarrow F$ such that $\Phi \leqslant \theta$ on $M$ and $\Phi=\varphi$ on $F$. Therefore, for every $x \in F$, we have $-\int_{A} \Psi(-x) d P \leqslant \Phi\left(x \chi_{A}\right) \leqslant \int_{A} \Psi(x) d P$ for all $A \in F$. Applying Corollary II.1 to the measiures $\mu_{x}:(\Omega, \mathscr{F}, P) \rightarrow E$ defined by $\mu_{x}(A)=\Phi\left(x \chi_{A}\right)$ gives a Bochner integrable random variable $f_{x}$ such that

$$
\mu_{x}(A)=\int_{A} f_{x} d P \quad \text { for every } A \in \mathscr{F} .
$$

It is immediate to check that the operator $T: F \rightarrow L^{1}[E]$ defined by $T(x)=f_{x}$ verifies the claimed properties.

Corollary II.3 was proved by M. Neumann in the case that $E$ has the RadonNikodym property [14].

Now let $\theta$ be a measure-preserving point transformation on $(\Omega, \mathscr{F}, P)$ and $I$ be the $\sigma$-field of $\theta$-invariant sets. If $E$ is a Banach space and $S$ an $E$-valued Bochner integrable random variable, the partial sums $S_{n}=\sum_{i-1}^{n} S \circ \theta^{i-1}$ form an additive process, that is we have for every $n, k \geqslant 0, S_{n+k}=S_{n}+S_{k} \circ \theta^{n}$. If $E$ is a Banach lattice and $\left(S_{n}\right)$ is a sequence of random variables satisfying for every $n, k \geqslant 0$, $S_{n+k} \leqslant S_{n}+S_{k} \circ \theta^{n}$, then $\left(S_{n}\right)$ is said to be a subadditive process.

The vector-valued ergodic theorem proved by Mourier in [13] asserts that $S_{n} / n=(1 / n) \sum_{i=1}^{n} S \circ \theta^{i-1}$ converges a.e. to $E^{I}[S]$. An alternate proof using the Kadec renorming theorem goes as follows: $\left\|S_{n}\right\|$ is a real-valued subadditive process, hence by Kingman's theorem [10], $\left\|S_{n}\right\| / n$ converges a.e. A standard 
argument shows that $S_{n}$ is Cauchy in $L^{1}[E]$ which permits us to identify the limit of $\left\|S_{n}\right\| / n$ as $\left\|E^{I}[S]\right\|$ and the theorem follows. A proof of this result using the Kadec-Klee renorming theorem also appears in [11].

THEOREM II.3 [7]. If $E$ is an order-continuous lattice, then for every positive $E$-valued subadditive process $\left(S_{n}\right)$ we have that $S_{n} / n$ converges strongly a.e.

Proof. Note first that if $\left(x_{n}\right)$ is a positive subadditive sequence $\left(x_{n+k} \leqslant x_{n}+x_{k}\right)$ then $\left(x_{2^{k}}\right) / 2^{k}$ is decreasing and $\inf \left(x_{n} / n\right)=\inf \left(x_{2^{k}} / 2^{k}\right)$. $\left\|x_{n}\right\|$ is a real subadditive sequence, thus $\lim \left(\left\|x_{n}\right\| / n\right)=\lim \left(\left\|x_{2^{k}}\right\| / 2^{k}\right)=\left\|\inf \left(x_{n} / n\right)\right\|$. Also, $f\left(x_{n}\right)$ is subadditive for every $f \in E_{+}$hence $f\left(x_{n} / n\right) \rightarrow f\left(\inf \left(x_{n} / n\right)\right)$. The norm convergence follows from the Kadec property of the norm.

Suppose now $\left(S_{n}\right)$ a positive subadditive process and $I$ the $\sigma$-field of $\theta$-invariant sets. Clearly, $\left(E^{I}\left[S_{n}\right](w)\right)$ is a subadditive sequence for almost every $w$, thus, if divided by $n$, it converges to $z(w)=\inf E^{I}\left(\left[S_{n}\right] / n\right)(w)$.

For every $f \in D$, we apply Kingman's theorem [10] to the real process $f\left(S_{n}\right)$ to obtain that $\lim f\left(S_{n} / n\right)=f\left(\inf E^{I}\left(\left[S_{n}\right] / n\right)\right)=f(Z)$ outside $\Omega_{f}$ with $P\left(\Omega_{f}\right)=0$. Therefore, $\lim \inf \left(\left\|S_{n}\right\| / n\right) \geqslant\|Z\|$ outside $\Omega_{0}=\cup_{f \in D} \Omega_{f}$.

Now fix $k$ and suppose $n \geqslant k$. Denote by $N(n)$ the integral part of $n / k$ and apply subadditivity to obtain

$$
S_{n} \leqslant \sum_{r=1}^{N(n)} S_{k} \circ \theta^{(r-1) k}+S_{n-N k} \circ \theta^{N R} \leqslant \sum_{r=1}^{N(n)} Y_{r}+W_{N}
$$

where $Y_{r}=S_{k} \circ \theta^{(r-1) k}$ and $W_{N}=\sum_{j=1}^{k-1} S_{j} \circ \theta^{N k}$. It follows that

$$
\lim \sup \frac{\left\|S_{n}\right\|}{n} \leqslant \lim \sup \frac{1}{N k}\left\|\sum_{r=1}^{N} Y_{r}\right\|+\lim \sup \frac{1}{N k}\left\|W_{N}\right\| .
$$

But $\sum_{r=1}^{N} Y_{r}$ is an additive process, hence, by Mourier's theorem, $(1 / N k) \sum_{r=1}^{N} Y_{r}$ converges to $E^{I}\left[S_{k} / k\right]$. On the other hand, $\left\|W_{N}\right\|$ has the same distribution for every $N$, thus by Borel-Cantelli, $\lim \left(\left\|W_{N}\right\| / N\right)=0$.

We get finally, $\lim \sup \left(\left\|S_{n}\right\| / n\right) \leqslant\left\|\inf E^{I}\left[S_{k} / k\right]\right\|$ a.e. and the renorming theorem gives the convergence.

\section{REFERENCES}

1. A. Bellow, Les amarts uniformes, C. R. Acad. Sci. Paris Sér. A-B 284 (1977), 1295-1298.

2. C. Bessaga and A. Pelczynski, Selected topics in infinite dimensional topology, PWN, Warsaw, 1975.

3. S. D. Chatterji, Martingale convergence and the Radon-Nikodym theorem in Banach spaces, Math. Scand. 22 (1968), 21-41.

4. M. Day, Normed linear spaces, 3rd ed., Ergebnisse der Mathematik und ihrer Grenzgebiete, Band 21, Springer-Verlag, Berlin and New York, 1973.

5. J. Diestel, Geometry of Banach spaces-selected topics, Lecture Notes in Math., vol. 485, SpringerVerlag, Berlin and New York, 1975.

6. J. Diestel and J. J. Uhl, Vector measures, Math. Surveys, no. 15, Amer. Math. Soc., Providence, R.I., 1977.

7. N. Ghoussoub and J. M. Steele, Vector valued subadditive processes and applications in probability, Ann. Probability (to appear).

8. H. Heinich, Convergence des sous-martingales positives dans un Banach recticule, C. R. Acad. Sci. Paris Sér. A-B, (1978). 
9. W. B. Johnson and L. Tzafriri, On the local structure of subspaces of Banach lattices, Israel J. Math. 20 (1975), 292-299.

10. J. F. C. Kingman, Subadditive processes, Lecture Notes in Math., vol. 539, Springer-Verlag, Berlin and New York, 1976, pp. 167-223.

11. A. Korzeniowski, A proof of the ergodic theorem in Banach spaces via amarts, Bull. Acad. Polon. Sci. 26 (1978), 1041-1044.

12. J. Lindenstrauss and L. Tzafriri, Classical Banach spaces-function spaces. II, Springer-Verlag, Berlin and New York, 1979.

13. E. Mourier, Elements aleatories dans un espace de Banach, Ann. Inst. H. Poincaré Sect. B 13 (1953), 161-244.

14. M. Neumann, On the Strassen disintegration theorem, Arch. Math. 29 (1977), 413-420.

15. J. Neveu, Discrete-parameter martingales, North-Holland Math. Library, Vol. 10, North-Holland, Amsterdam and Oxford; American Elsevier, New York, 1975.

16. H. H. Schaefer, Banach lattices and positive operators, Springer-Verlag, Berlin and New York, 1975.

Department of Mathematics, Ohio State University, Columbus, Oho 43210

Department of Mathematics, University of British Columbia, Vancouver V6T 1W5, British Columbia, Canada

Mathematics Institute, The Hebrew University, Jerusalem, IsRael 